\title{
Symmetry Energy Dependence of Light Fragment Production in Heavy Ion Collisions
}

\author{
H.H. Wolter ${ }^{1, a}$, M. Zielinska-Pfabe ${ }^{2}$, P. Decowski ${ }^{2}$, M. Colonna ${ }^{3}$, R. Bougault ${ }^{4}$, and A. Chbihi ${ }^{5}$ \\ ${ }^{1}$ Fac. of Physics, University of Munich, D-85748 Garching, Germany \\ ${ }^{2}$ Smith College, Northampton, Mass. 01063, USA \\ ${ }^{3}$ INFN, Laboratori Nazionali del Sud, , I-95123, Catania, Italy \\ ${ }^{4}$ LPC Caen, Univ. de Caen, F-14050 Caen, France \\ ${ }^{5}$ GANIL, F-14076 Cedex, France
}

\begin{abstract}
We study the pre-equilibrium light cluster emission in low to intermediate energy heavy ion collisions in transport simulations as a means to obtain information about the density and momentum dependence of the nuclear symmetry potential, i.e. about the asy-stiffness and the neutron-proton effective mass splitting of the symmetry energy. We find that the study of $n / p$ and $t /{ }^{3} \mathrm{He}$ ratios as a function of the energy of the emitted particles may allow to disentangle these two behaviors The $t /{ }^{3} \mathrm{He}$ ratios appear to carry similar information as the $n / p$ ratios, making this a promising option for experimental investigations. More $n$-rich systems enhance the sensitivity to the symmetry energy, while double ratios between $n$-rich and $n$-poor systems tend to reduce it. A preliminary comparison to experimental data for the ${ }^{136,124} \mathrm{Xe}+{ }^{124,112} \mathrm{Sn}$ systems is also shown.
\end{abstract}

The density dependence of the nuclear symmetry energy (NSE) is of large actual interest in nuclear physics with important implications in astrophysics, particularly in the properties of neutron stars. It is not well determined in microscopic calculations, and therefore has been investigated via nuclear structure observables and in heavy ion collisions. The advantage of the latter is that by choosing different collision systems, incident energies and impact parameters one can access different densities and asymmetries of nuclear matter.

In this contribution we consider nuclear matter around and below saturation densities in collision with energies from the Coulomb barrier to a few $100 \mathrm{MeV}$ per particle. In this regime the decomposition of the system into fragments is the main decay mode. From the theoretical point of view isospin transport and fractionation play the essential role in the decay of the system, which depend on the properties of the NSE. It is customary to refer to a density dependence with a large slope at saturation as asy-stiff, and to the opposite situation as asy-soft. A number of observables have been identified as sensitive to the NSE. One is the pre-equilibrium emission of nucleons and light fragments in the initial stages of the collision, which depends directly on the neutron and proton potentials. To enhance as much as possible the sensitivity to the NSE one considers ratios or differences of observables of isobaric pairs of particles, such as neutrons to protons, tritons to ${ }^{3} \mathrm{He}$ or $\pi^{-}$to $\pi^{+}$for isotopic yields, but also for flow observables.

\footnotetext{
ae-mail: hermann.wolter@lmu.de
} 
The most direct evidence of the NSE thus is expected in the neutron to proton ratios. In fact, this has been observed in medium energy collisions of different $\mathrm{Sn}$ isotopes [1]. Since for a $n$-rich medium the neutron potential is repulsive below saturation, and more so for an asy-soft NSE, the neutron over proton ratio should be larger for an asy-soft NSE, which was also the conclusion of the above analyses. An experimental difficulty is that neutron and protons are measured with different phase space coverage. To reduce these uncertainties of neutron-to-proton ratios between systems of different asymmetry, so-called double ratios, were considered. However, in double ratios some of the sensitivity to the detailed features of the symmetry energy may be lost.

It is thus of interest to consider ratios of light charged particles, in particular $t /{ }^{3} \mathrm{He}$, since the experimental uncertainties are reduced. Indeed, this has been studied theoretically mostly at higher energies of several hundred MeV/A for yield ratios and flows [2], but no data exist until now. The present study is directed to a systematic theoretical study of $t /{ }^{3} \mathrm{He}$ yield ratios, motivated by experimental data of the system ${ }^{136,124} \mathrm{Xe}+{ }^{124,112} \mathrm{~S} n$ at energies of $\mathrm{E} / \mathrm{A}=32$ to $150 \mathrm{MeV}$.
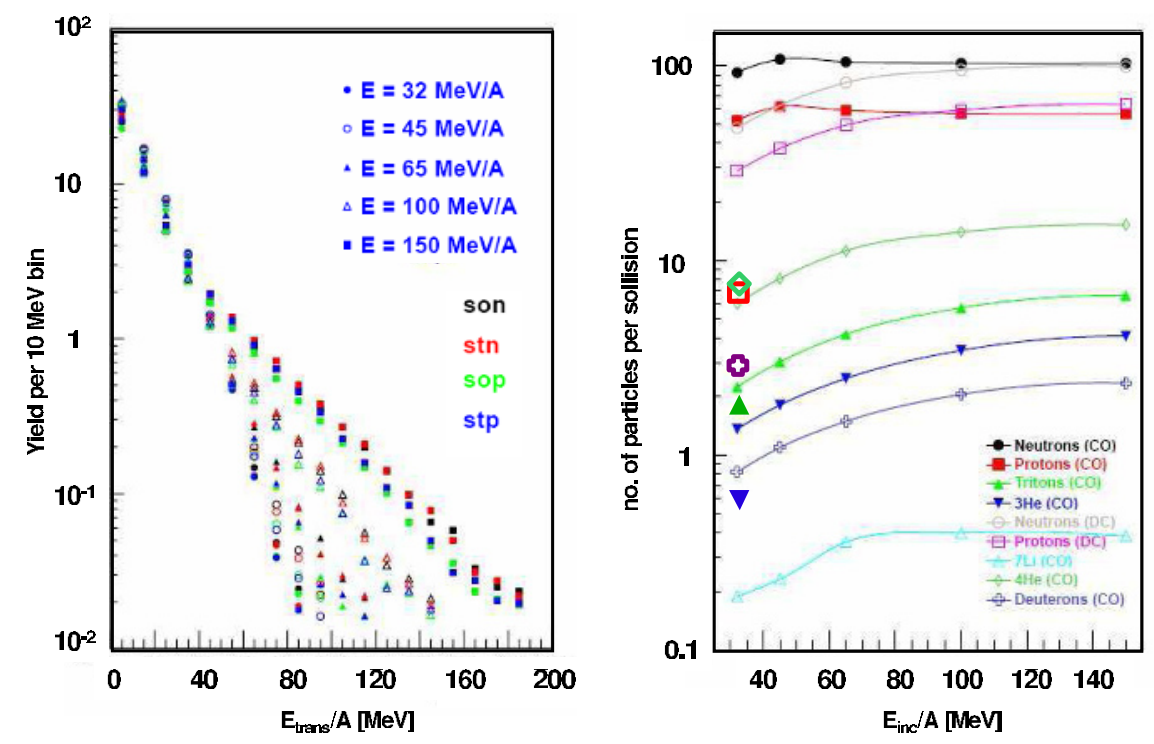

Figure 1. (left) Proton transverse energy spectra for different incident energies (symbols identified in the figure) and different model discussed in the text (colors); (right) Total yields of different particles (identified in the figure) as a function of the incident energy for model "son"; $n$-rich system in both panels.

In general, the isospin-dependent mean field depends on density, but also on momentum, which arises from exchange and correlation contributions. This is important in heavy ion collisions where the particles may have high momenta. The momentum dependence of the isovector potential can be expressed in terms of an effective mass as $m_{i} * / m=\left(1+\left(m / \hbar^{2} k\right) \partial U_{i} / \partial k\right)^{-1} ; i=(n, p)$, and thus leads to a splitting of the effective masses between protons and neutrons. The magnitude and even the sign of this splitting is controversial in many-body calculations and in analyses of heavy ion collisions [3].

We present results of transport calculations for the $n$-rich system ${ }^{136} X e+{ }^{124} S n$ and the $n$-poor system ${ }^{124} \mathrm{Xe}+{ }^{112} \mathrm{Sn}$ at energies between 32 and $150 \mathrm{AMeV}$. The simulations are performed with the Stochastic Mean Field (SMF) simulation code of the transport equations, which is a BUU-type 
implementation with an approximation to a fluctuation term. The mean field is parametrized as a Bombaci-Gale-Bertsch-DasGupta (BGBD)-type potential which allows to change the density- and momentum dependence of the symmetry potential without changing the isoscalar behavior [4]. We perform calculations with all four combinations of asy-soft and -stiff density dependence and $m_{n}^{*}>m_{p}^{*}$ and $m_{p}^{*}>m_{n}^{*}$, denoted as son, sop, stn, stp, respectively. Clusters are identified by coalescence at the freeze-out time, where we employ two methods: $(\mathrm{CO})$ coalescence in phase space with coalescence radii of $r_{0}=1.6 \mathrm{fm}$ and $p_{0}=1.3 \mathrm{fm}^{-1}$; (DC) density-cut in coordinate space with $\rho>0.03 \mathrm{fm}^{-3}$ as the "liquid" and everything else as a gas of neutrons and protons. In the present results we consider central collisions with impact parameter of $b=2 \mathrm{fm}$.

In Fig. 1 we show in the left panel proton transverse energy spectra for different energies and the different models son, sop, stn, stp referred to above. We see the generally exponential decay of the spectra, but also the (relatively small) influence of the models. On the right we display the total yields per collision for the different light particles up to ${ }^{7} \mathrm{Li}$. Protons and neutrons are shown for both the phase space coalescence (CO) and the density cut (DC) methods of fragment recognition. It is seen that the $\mathrm{CO}$ procedure produces too many nucleons relative to the DC method, which was seen to compare generally well with data [5]. For $E_{i n c}=32 \mathrm{AMeV}$ we show by symbols the total yield of preliminary experimental data. It is seen that nucleons are substantially, and that tritons and ${ }^{3} \mathrm{He}$ 's are somewhat overproduced, while deuterons are underproduced in the simulations. The production of alpha particles is about correct, and is larger than that of the $\mathrm{A}=3$ particles, showing that the mean field begins to give some binding to alpha clusters. The experimental data include particles from the deexcitation of the primary fragments while the calculations do not. Thus this comparison is qualitative, but eventually it should e possible to identify the evaporative component in the low energy spectra. Also it is hoped that the ratios of cluster yields, as studied here, are still meaningful, and in particularly less sensitive to the evaporation component.

In Fig. 2 we show various single and double ratios for the different models. In the upper row we compare the $n / p$ single ratios for the $n$-rich system at energies of (a) 32 and (b) $150 \mathrm{AMeV}$, and (c) of the $n$-poor system at $150 \mathrm{AMeV}$. It is seen that the difference between the models is more pronounced at the higher energy and the $n$-rich system. A very interesting behavior of these single ratios on the density (asy-soft or -stiff) and momentum $\left(m_{n}^{*}>m_{p}^{*}\right.$ or $\left.m_{p}^{*}>m_{n}^{*}\right)$ dependence is observed. It is most clearly seen in panel (b) for the $n / p$ ratio in the $n$-rich system at the higher energy: The ratio at low transverse energies is dominated by the asy-stiffness of the NSE, since the two models with an asy-soft dependence (green and black), respectively an asy-stiff dependence (red and blue) behave similarly. At high momentum, on the other hand, the two models with $m_{p}^{*}>m_{n}^{*}$ (green and blue) and with $m_{n}^{*}>m_{p}^{*}$ (black and red), respectively have similar slopes. This is an indication, that the behavior of the ratios can give the very interesting possibility to separate the density and the momentum behavior of the symmetry potential. This behavior is also seen but less strongly in the $n$-poor system (panel (c)), as one would expect. In panel (d) we see that the corresponding $t /{ }^{3} \mathrm{He}$ ratios behave in a similar way as the $n / p$ ratios. It is not so clearly seen in the figure, since the $t$ and ${ }^{3} \mathrm{He}$ spectra are cut off at too low energies due to the statistics of the simulations, as mentioned above. When this is improved it is expected that they show similar characteristics as the $n / p$ ratios.

The double ratios are shown in panels (e) for $n / p$ and (f) for $t /{ }^{3} \mathrm{He}$ at the higher energy. It is generally seen that the double ratios are considerably less sensitive to the models for the NSE. The dependence on the stiffness at the lower transverse energies has practically disappeared and the dependence on the effective mass is much smaller; compare e.g.panels (b) and (e) for the $n / p$ and panels (d) and (f) for the $t /{ }^{3} \mathrm{He}$ ratios. Thus in the double ratios a considerable part of the sensitivity to the characteristics of the NSE is lost. The consideration of single ratios seems preferable, esp. when there is no strong experimental argument against it, as for the $t /{ }^{3} \mathrm{He}$ ratios. The (single) ratio of 
neutrons for the $n$-rich over the $n$-poor system (not shown) behave very similarly to the corresponding $n / p$ double ratio, since the proton yields for the two systems with identical proton content are very similar and thus cancel out to a large extent.
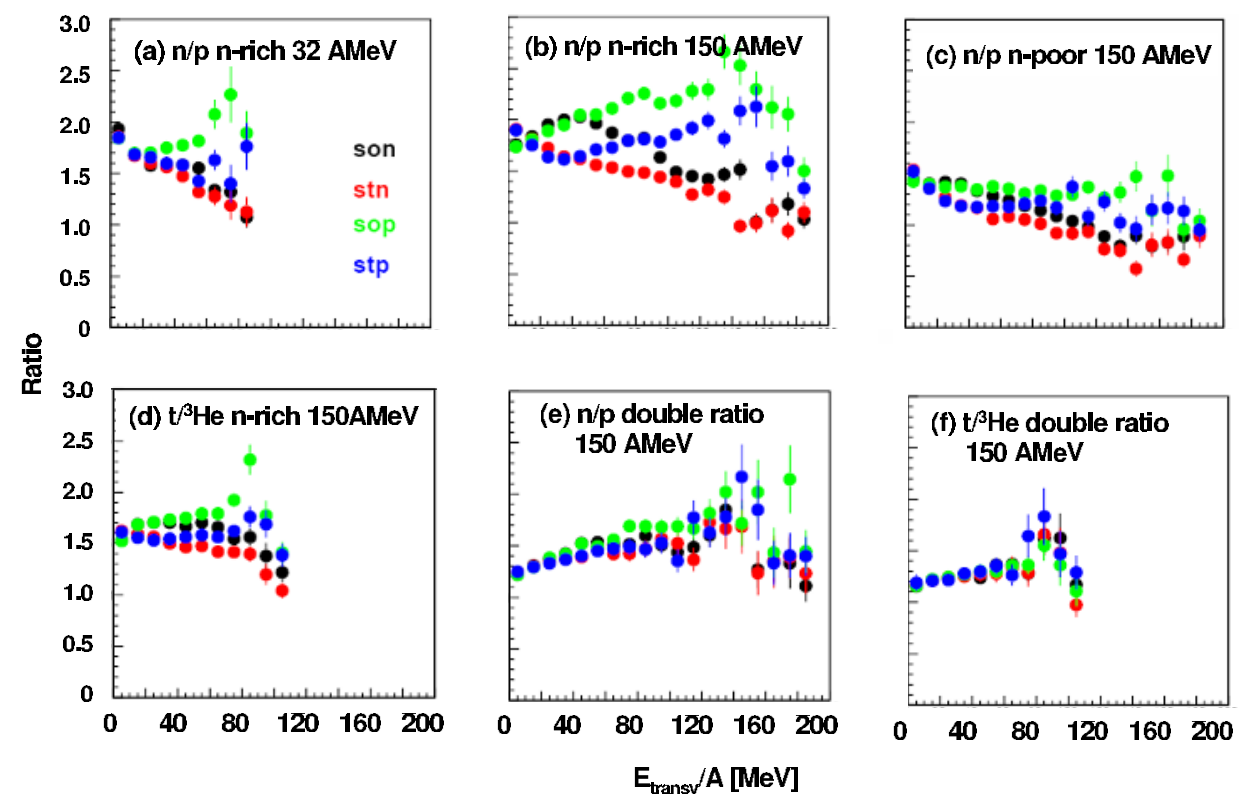

Figure 2. Single and double ratios of light emitted particles as a function of transverse energy per nucleon for the different models (colors, see text). Upper row: $n / p$ single ratios for the $n$-rich system for incident energies of (a) $32 \mathrm{AMeV}$, (b) $150 \mathrm{AMeV}$, and (c) for the $n$-poor system at $150 \mathrm{AMeV}$; lower row: (d) $t /{ }^{3} \mathrm{He}$ ratio for the $n$-rich system at $150 \mathrm{AMeV}$; double ratios for (e) $n / p$ and (f) $t /{ }^{3} \mathrm{He}$ both at $150 \mathrm{AMeV}$.

In summary, the present study shows that ratios of yields of isobaric pairs in pre-equilibrium cluster emission can be a useful tool to learn about the density and momentum dependence of the symmetry potential. In particular, the dependence of these ratios as a function of the emission transverse energy appears as a way to disentangle the density and momentum dependence. It is seen that $t /{ }^{3} \mathrm{He}$ ratios carry much the same information as $n / p$ ratios, and thus represent a promising experimental option. We also find, that double ratios tend to reduce the information on the detailed dependence of the NSE. A study of flow observables will complement this study in the future, as well as a more detailed comparison with experiment.

This work was supported in part by the Universe-cluster of excellence of the DFG, Germany.

\section{References}

[1] M. Famiano, et al., PHys. Rev. Lett. 97, 052701 (2006)

[2] G.C.Yong, et al.,Phys. Rev. C80, 044608 (2009); V. Giordano, et al.,Phys. Rev. C81, 044611 (2010); Z.Q. Feng, Nucl. Phys. bA878, 3 (2012)

[3] Bao-An Li, et al., arXiv:1304.3368

[4] V. Baran, et la., Phys. Rep. 410, 335 (2005)

[5] S. Houdan, et al., Phys. Rev. 67, 064613 (2003) 